\title{
Statistical Shape Analyses of Patients with Scaphocephaly After Surgical Remodeling
}

\author{
Gokhan OCAKOGLU', Mevlut Ozgur TASKAPILIOGLU², Seckin KAYA², Zeynep YAZICl ${ }^{3}$ \\ ${ }^{1}$ Uludag University, School of Medicine, Department of Biostatistics, Bursa, Turkey \\ 2Uludag University, School of Medicine, Department of Neurosurgery, Bursa, Turkey \\ ${ }^{3}$ Uludag University, School of Medicine, Department of Radiology, Bursa, Turkey \\ Corresponding author: Gokhan OCAKOGLU gocakoglu@gmail.com
}

\section{ABSTRACT}

AIM: To investigate the differences in the head shapes of scaphocephaly patients during the pre- and postoperative phases.

MATERIAL and METHODS: Cranium shape data were collected from the two-dimensional digital images. The generalised Procrustes analysis was used to obtain mean shapes in the pre- and postoperative phases. The shape deformation of the cranium from the pre- to postoperative phases was evaluated using the thin plate spline method.

RESULTS: There was no significant cranial shape difference in the pre and postoperative phases. The high-level deformations from the pre- to postoperative phase were seen in thin plate spline graphs. The highest deformation was observed at the biparietal dimension.

CONCLUSION: In this study, we showed that the structural deformation of the cranium was correlated with sagittal craniosynostosis. The present study also shows that pre- and postoperative head shapes of patients with scaphocephaly can be compared using head shape with the landmark-based geometric morphometric method by taking into consideration the topographic distribution.

KEYWORDS: Sagittal craniosynostosis, Cranium, Head shape, Statistical shape analysis

\section{INTRODUCTION}

C raniosynostosis is a congenital malformation that occurs when there is premature ossification of one or more sutures of a newborn's skull. Craniosynostosis can result in abnormal head shape, visual impairment, intracranial hypertension and intellectual impairment (11).

The most common single-suture non-syndromic craniosynostosis is scaphocephaly, which is the premature closure of the sagittal suture. This occurs in $50 \%-60 \%$ of all cases (18). The incidence of scaphocephaly is $1 / 5000$ children with a male predominance of 4:1 ratio (18).

Standard procedure for patients with scaphocephaly is surgery, which aims to correct the cranial dysmorphia aside from its cosmetic benefits. The most common operation is barrel-stave osteotomies and cranial remodelling for scaphocephaly (9).
Many areas of medicine are related to the examination of the geometric feature of the organ or organism. In these studies, it is possible to work with the geometric shapes by taking the coordinates of the landmark points as the variables in the figure from the measurements taken from the organisms $(1,12-14,17)$.

Many different methods based on volume, area and shape measurements have been used to measure changes preand postoperative cranial vault $(3,21)$. With the technological advancementin medicine, various methods have been developed based on the calculation of the geometric places of particular landmarks for the analysis of the shapes of the organs. Statistical shape analysis is a modern geometric morphometric method that uses the shape of organs or organisms as input data.
Gokhan OCAKOGLU (1) : 0000-0002-1114-6051

Mevlut Ozgur TASKAPILIOGLU (10) : 0000-0001-5472-9065
Seckin KAYA (D): 0000-0002-4256-2250

Zeynep YAZICI (1) : 0000-0002-8647-5298 
This study aimed to investigate head shape differences of scaphocephaly patients in the pre- and postoperative phases.

\section{MATERIAL and METHODS}

\section{Subjects}

This study was conducted using the computed tomography (CT) scans of 20 (12 males, eight females) scaphocephaly patients aged between 3 and 75 months (median=9.50). The CT scans of scaphocephaly patients admitted to the Uludag University Faculty of Medicine Department of Neurosurgery, between March 2004 and November 2017, were evaluated retrospectively. All CT imaging examinations performed on a multi-slice CT scanner (SOMATOM Plus 4, Siemens Medical Solutions, Forchheim, Germany). All CT scans were obtained

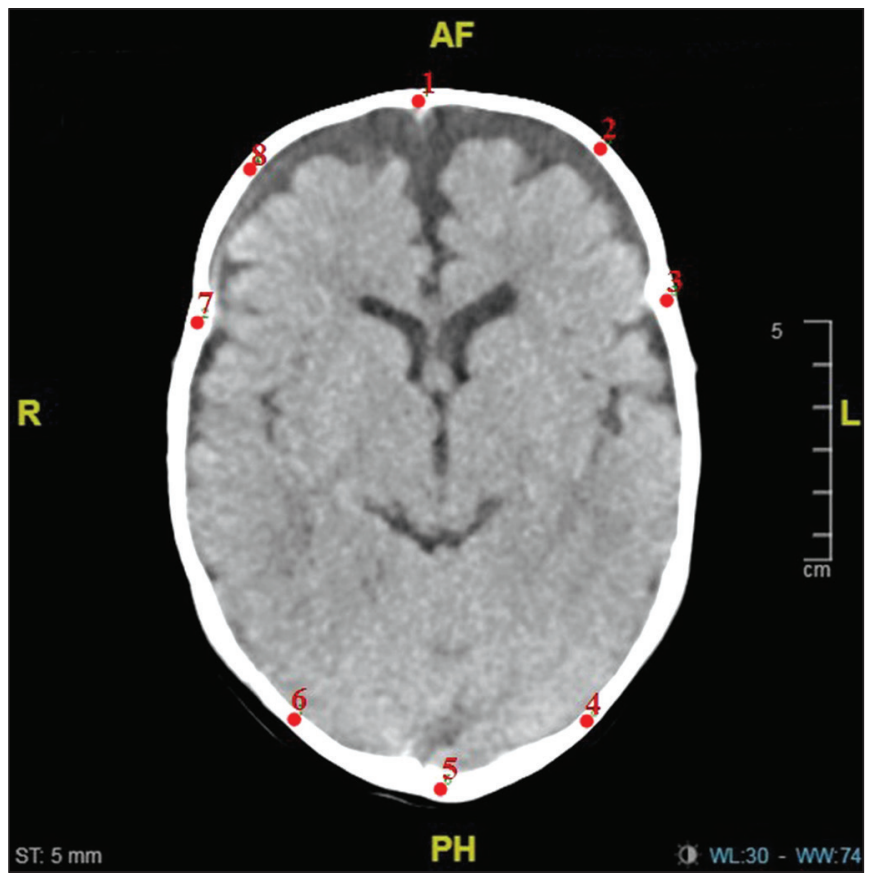

Figure 1: Landmark markings on the cranium. at the first postoperative day. All operations were performed by the same surgeon in the Department of Neurosurgery, Faculty of Medicine, at Uludag University (MOT). Informed consent was obtained from all participants, and the university ethics committee approved this prospective study and the study protocol.

\section{Collection of Two-Dimensional Cranial Landmarks}

We implemented a statistical shape analysis by using homologous anatomic landmarks, which are selected as the most relevant. Eight cranial landmarks corresponding to the foramen of Monro were selected from the pre- and postoperative images (Figure 1). The descriptions of these landmarks are given in Table I. The landmarks were chosen based on reliability, maximising anatomic coverage and cranial morphologic descriptions. Cranial data were collected from the two-dimensional digital images. Standard anatomic landmarks were selected and were marked on each digital image using TPSDIG 2.04 software (15).

\section{Geometric Morphometric Analysis}

Statistical Shape Analysis: Statistical shape analysis of the pre- and postoperative cranial images of scaphocephaly patients was performed. The generalised Procrustes analysis was used to obtain the mean cranium shape in the pre- and postoperative phases and related tangent coordinates. Procrustes analysis is known as the approach that obtains the minimum sum of squared differences between the landmarks (5). Principal coordinate analysis (PCoA), which is a kind of ordination method, also known as metric multidimensional scaling was applied to tangent coordinates that were derived from the Procrustes analysis. The PCoA routine finds the eigen values and eigenvectors of a matrix containing the distances or similarities between all landmark points. Euclidean distance is used in PCoA. The eigen values, giving a measure of the variance accounted for by the corresponding eigenvectors (coordinates), were reported. The percentages of variance accounted for by these components are also reported. After that, classification and changes of scaphocephaly patients were examined for pre- and postoperative time points using

Table I: Definitions of Landmarks Used in the Present Study

\begin{tabular}{ll}
\hline Landmark & Definition \\
\hline 1 & Frontmost point of the cranium \\
\hline 2 & $\begin{array}{l}\text { The point at which the line that passes through the midpoint of the segment, which was drawn from } \\
\text { landmark } 1 \text { to landmark } 3\end{array}$ \\
\hline 3 & Coronal suture \\
\hline 5 & Lambdoid suture \\
\hline 6 & Backmost point of the cranium \\
\hline 7 & Lambdoid suture \\
\hline 8 & Coronal suture \\
\hline
\end{tabular}


PCoA. The mean of the head shapes obtained by Procrustes analysis during the pre- and postoperative periods was compared using the paired Hotelling $\mathrm{T}^{2}$ test. The shape deformation of the cranium from the pre- to postoperative phases was evaluated using the thin plate spline (TPS) method, which was derived from a mathematical model used in computer graphics and applied to morphometrics by Bookstein (2). The points exhibiting the greatest enlargements or reductions labelled as deformations were established through the TPS analysis.

Landmark Reliability: Reliability coefficient (G) for a twofacet crossed design ('landmark pairs-by-rater-by-subject') based on the generalisability theory was calculated (6).

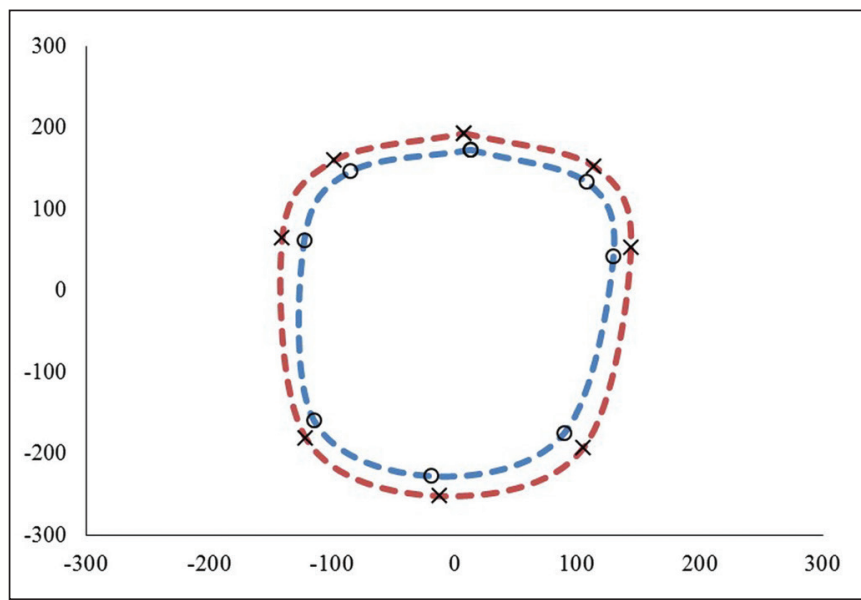

Figure 2: Procrustes mean shapes for the pre- and postoperative phases of cranium images of scaphocephaly patients (o: Preop and $\mathbf{x}$ : Postop).
The same researcher marked all the landmarks. To be able to compute for $G$ coefficient, the same researcher again marked 10 subjects randomly selected from the participants 2 weeks later. The values of the $\mathrm{G}$ coefficient reflected strong repeatability $(\mathrm{G}=0.978)$.

In this study, R 3.5.1 (20), PAST 3.0 (10) and SPSS (19) softwares were used for statistical analysis.

\section{RESULTS}

Procrustes mean shapes (Figure 2) obtained as a result of landmark markings from the pre- and postoperative periods of patients with scaphocephaly were compared with paired Hotelling $\mathrm{T}^{2}$ test, and no difference in shape was found between the pre- and postoperative phases $(p=0.658)$. PCoA was applied to the tangent coordinates obtained after Procrustes analysis. PCoA of the pre- and postoperative phases showed variance explanation rate of $75.88 \%$ for the first two coordinates considered in scaphocephaly patients (Figure 3). The scatter plot graph (Figure 4) obtained from PCoA analysis was plotted according to the first two eigen values that explained the most variance rate. The 95\% confidence ellipses and convex hulls on the scatterplot indicate that the results did not differ significantly in the pre- and postoperative periods, and variability in the pre- and postoperative periods was not high. It supports the claim that there is no difference between the pre- and postoperative mean patterns obtained after the paired Hotelling $\mathrm{T}^{2}$ test.

TPS analysis was also performed using mean shapes obtained from the Procrustes analysis. A high level of deformations of the cranium from the pre- to postoperative phases was observed in the TPS graph (Figure 5). The highest deformation

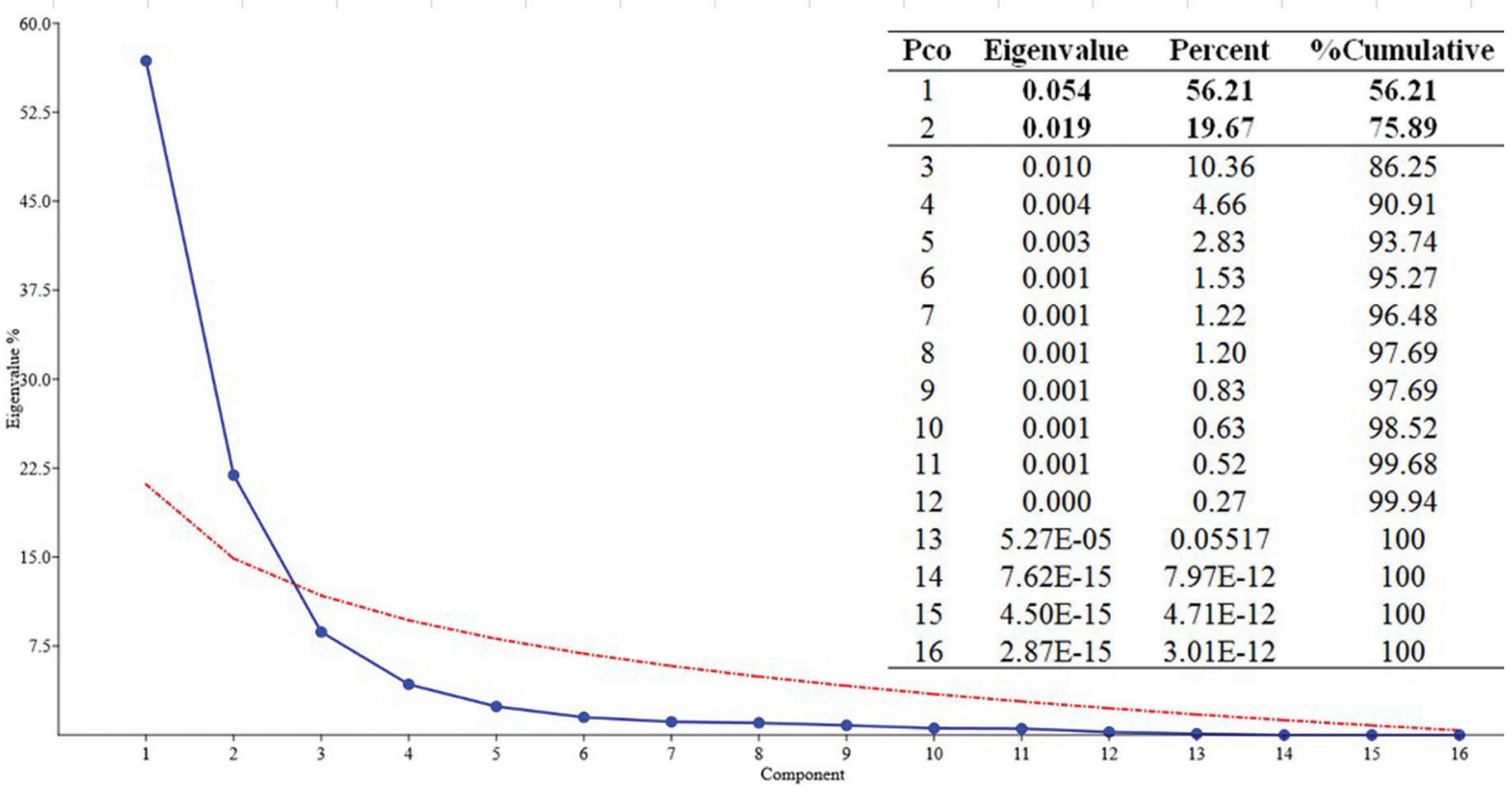

Figure 3: A screen plot of the percentage variability explained by each principal coordinate. 

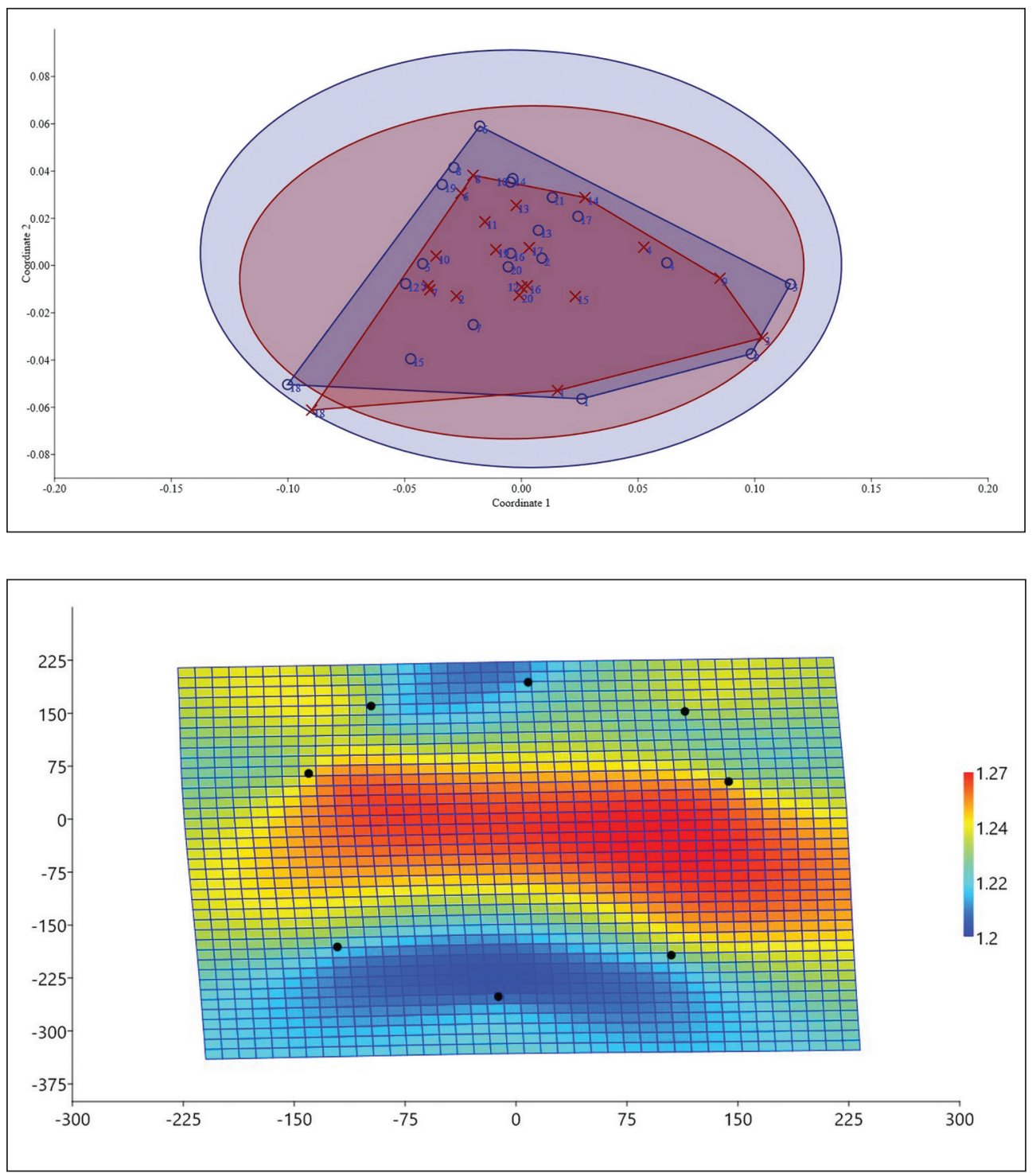

Figure 4: Scatter graphs with 95\% confidence ellipses and convex hulls obtained by principal coordinate analysis results of 20 scaphocephaly patients (o: Preop, x: Postop).
Figure 5: A thin plate spline demonstrating the average cranium shape deformation for preoperative phase compared with postoperative phase. was found in the biparietal dimension shown in Figure 5. When the mean shapes and the deformation were taken into consideration, based on the pre- to postoperative periods, it is observed that there is generally an expansion in the shape of the head.

\section{DISCUSSION}

Craniosynostosis was defined by Virchow in 1859 as early the closure of the sutures followed by secondary skull deformities (4). Scaphocephaly is the most common isolated type of synostosis with an incidence of 56\%-58 \% (4).

We performed a statistical shape analysis to show the change in the cranium in the pre- and postoperative periods in patients with scaphocephaly. In addition to the general changes, the landmark-based shape analysis approach showed us the changes on a regional basis. Since statistical shape analysis allows the preservation of morphologic, the main reasons why statistical shape analysis is widely used in medicine are advances in imaging technology, and it is also used to investigate the effects of diseases and the effects of environmental factors on the structure of the organ or organism (7).

Sharma et al. mentioned an increase in the mean cephalic index $(\mathrm{Cl})$ from 0.64 to 0.75 , and the mean intracranial volume increased from $1481 \mathrm{~cm}^{3}$ preoperatively to $1671 \mathrm{~cm}^{3}$ postoperatively (16). But these changes were global; the authors did not mention the direction of expansion. Our results support the Fearon et al.'s results suggesting that the $\mathrm{Cl}$ is not a reliable outcome measure because of the inefficacy to realise the amount of correction (8). We analysed the shape of the cranium in scaphocephaly patients in the pre- and postoperative phases in this study. There was no statistical difference between the pre- and postoperative periods in terms of the mean head shape.

Although there was no difference between the mean shapes, deformation was determined in the preoperative period. 
Deformation is generally in the direction of expansion. However, most deformations are observed in the area delimited by landmark 3, 4 and landmark 6, 7 at the postoperative period. The postoperative images that were used in this study were immediate images of the patients that were obtained during the early postoperative period. The expansion detected at the landmark 3, 4 and landmark 6, 7 showed the expansion of the width of the cranium, demonstrating the efficiency of the operation.

In this study, we showed that the structural deformation of the cranium was correlated with the sagittal craniosynostosis. These results are supported by previous morphologic studies that also show in some cases. The present study and previous literature suggest that additional studies using more control groups of sagittal craniosynostosis patients are needed to further understand this pathology and design new treatment strategies.

The main limitation of this study is the limited patient number. A difference at the mean head shape could be determined between the pre- and postoperative periods of scaphocephaly patients if the number of patients was higher.

\section{CONCLUSION}

This study showed that shape deformations were observed in the cranium in sagittal craniosynostosis patients. To the best of our knowledge, this is the first study to show that pre- and postoperative head shapes of patients with scaphocephaly can be compared using head shape with the landmark-based geometric morphometric method by taking into consideration the topographic distribution. We hope that the results obtained from this study serve as a guide to future clinical studies by demonstrating localised variations in the components of the scaphocephaly that constitute the overall shape of the scaphocephaly in sagittal craniosynostosis patients.

\section{- REFERENCES}

1. Baskan EB, Ercan I, Ozdemir ST, Ozkaya G, Yilmaz M, Ozgenel $\mathrm{Y}$, Saricaoglu $\mathrm{H}$ : A case of parry-romberg syndrome: Examining the efficacy of lipofilling by statistical shape analysis. Turkiye Klinikleri J Med Sci 33:284-289, 2013

2. Bookstein FL: Principal warps: Thin-plate splines and the decomposition of deformations. IEEE Transactions on Pattern Analysis and Machine Intelligence 11:567-585, 1989

3. Calandrelli R, Pilato F, Massimi L, Panfili M, Di Rocco C, Colosimo C: Quantitative analysis of cranial-orbital changes in infants with anterior synostotic plagiocephaly. Childs Nerv Syst 34(9):1725-1733, 2018

4. Ciurea A, Toader C, Mihalache C: Actual concepts in scaphocephaly: An experience of 98 cases. J Med Life 4(4):424-431, 2011

5. Dryden IL, Mardia KV: Statistical shape analysis: With applications in R. 2nd ed. John Wiley \& Sons, 2016
6. Ercan I, Ocakoglu G, Guney I, Yazici B: Adaptation of generalizability theory for inter-rater reliability for landmark localization. International Journal of Tomography \& Statistics 9:51-59, 2008

7. Ercan I, Ocakoglu G, Sigirli D, Ozkaya G: Statistical shape analysis and usage in medical sciences. Turkiye Klinikleri Journal of Biostatistics 4:27-35, 2012

8. Fearon JA, Ditthakasem K, Herbert M, Kolar J: An appraisal of the cephalic index in sagittal craniosynostosis, and the unseen third dimension. Plast Reconstr Surg 140:138-145, 2017

9. Ginat DT, Lam D, Kuhn AS, Reid R: CT imaging findings after craniosynostosis reconstructive surgery. Pediatric Neurosurgery 53:215-221, 2018

10. Hammer $\varnothing$, Harper D, Ryan P: Paleontological statistics software: Package for education and data analysis. Palaeontologia Electronica 4(1):1-9, 2001

11. Meulstee JW, Verhamme LM, Borstlap WA, Van der Heijden F, De Jong GA, Xi T, Bergé SJ, Delye H, Maal TJJ: A new method for three-dimensional evaluation of the cranial shape and the automatic identification of craniosynostosis using 3D stereophotogrammetry. Int J Oral Maxillofac Surg 46:819-826, 2017

12. Ocakoglu G, Ercan I: Traditional and modern morphometrics. Turkiye Klinikleri Journal of Biostatistics 5(1):37-41, 2013

13. Ocakoglu G, Ozdemir ST, Ercan I, Etoz A: The shape of the external human ear: A geometric morphometric study. Turkiye Klinikleri J Med Sci 33:184-190, 2013

14. Ocakoglu G, Taskapilioglu MO, Ercan I, Demir AB, Hakyemez B, Bekar A, Bora I: Statistical shape analysis of temporal lobe in mesial temporal sclerosis patients. Acta Neurochirurgica 157:1897-1903, 2015

15. Rohlf FJ: Tps Dig, ver. 2.04. Department of Ecology and Evolution. State University of New York at Stony Brook, 2005

16. Sharma JD, O'Hara JL, Borghi A, Rodriguez-Florez N, Breakey W, Ong J, Jeelani NO, Dunaway DJ, James G: Results following adoption of a modified Melbourne technique of total scaphocephaly correction. Journal of Craniofacial Surgery 29:1117-1122, 2018

17. Sigirli D, Ercan I: Growth and allometry in modern morphometrics. Turkiye Klinikleri Journal of Biostatistics 5(1):42-48, 2013

18. Simpson A, Wong AL, Bezuhly M: Surgical correction of nonsyndromic sagittal craniosynostosis: Concepts and controversies. Annals of Plastic Surgery 78:103-110, 2017

19. SPSS: Statistics for Windows, version 20.0. IBM Corp., Armonk, NY, 2012

20. Team RC: R: A language and environment for statistical computing. R Foundation for Statistical Computing, Vienna, Austria. R Development Core Team, 2014

21. Ter Maaten NS, Mazzaferro DM, Wes AM, Naran S, Bartlett SP, Taylor JA: Craniometric analysis of frontal cranial morphology following posterior vault distraction. J Craniofac Surg 29:11691173, 2018 